Provided for non-commercial research and education use. Not for reproduction, distribution or commercial use.

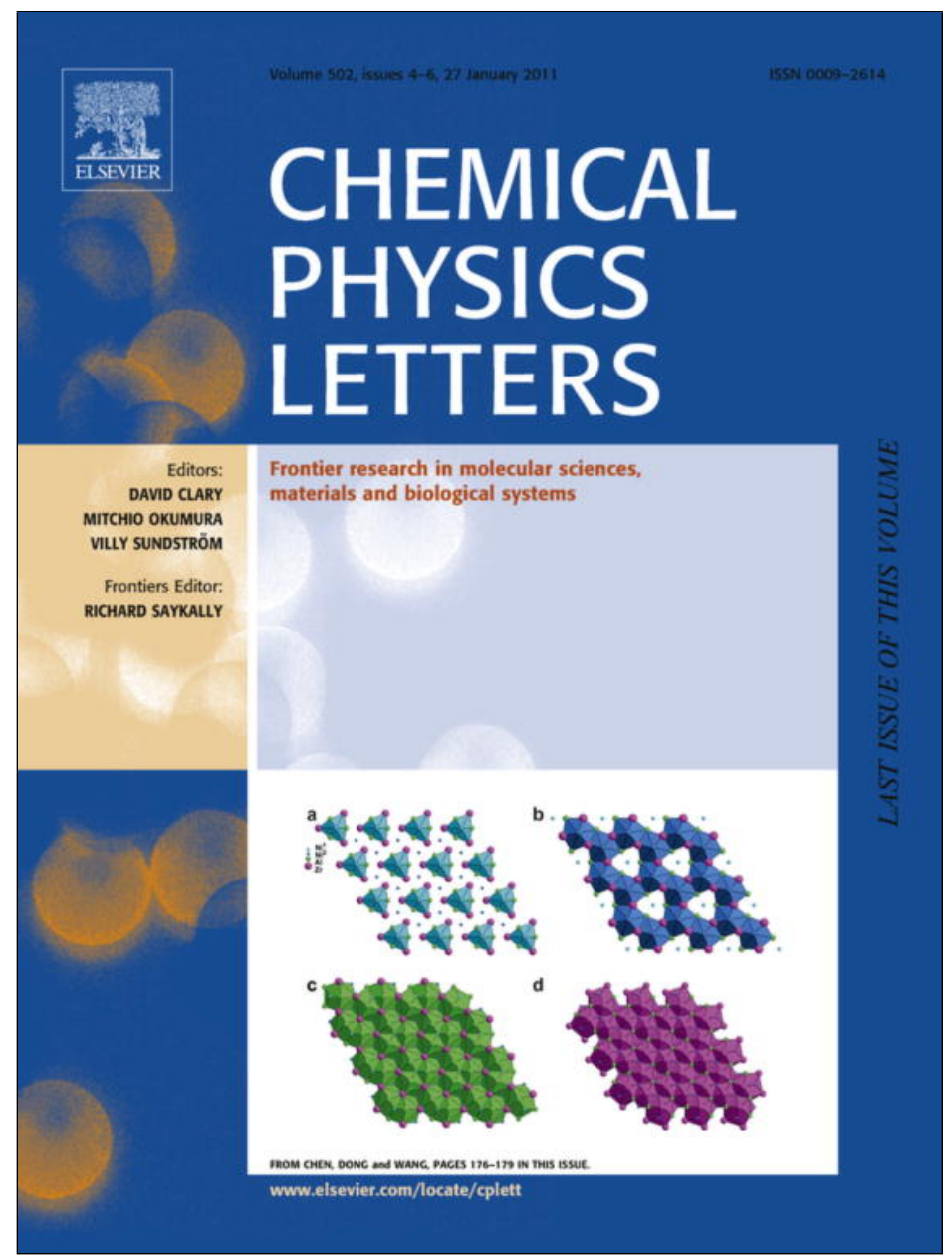

This article appeared in a journal published by Elsevier. The attached copy is furnished to the author for internal non-commercial research and education use, including for instruction at the authors institution and sharing with colleagues.

Other uses, including reproduction and distribution, or selling or licensing copies, or posting to personal, institutional or third party websites are prohibited.

In most cases authors are permitted to post their version of the article (e.g. in Word or Tex form) to their personal website or institutional repository. Authors requiring further information regarding Elsevier's archiving and manuscript policies are encouraged to visit:

http://www.elsevier.com/copyright 


\title{
Probing the quenching of rotary resonance by PISSARRO decoupling
}

\author{
Markus Weingarth $^{\text {a,b,c,1 }}$, Geoffrey Bodenhausen ${ }^{\mathrm{a}, \mathrm{b}, \mathrm{c}}$, Piotr Tekely ${ }^{\mathrm{a}, \mathrm{b}, \mathrm{c}, *}$ \\ ${ }^{a}$ Ecole Normale Supérieure, Département de Chimie, 24 rue Lhomond, 75005 Paris, France \\ ${ }^{\mathrm{b}}$ Université Pierre et Marie Curie, 4, Place Jussieu, 75005 Paris, France \\ ' CNRS, UMR 7203, Département de Chimie, 24 rue Lhomond, 75005 Paris, France
}

\section{A R T I C L E I N F O}

\section{Article history:}

Received 15 October 2010

In final form 11 December 2010

Available online 15 December 2010

\begin{abstract}
A B S T R A C T
Solid-state NMR experiments may suffer from rotary resonance recoupling $\left(R^{3}\right)$ due to the interference between sample spinning at a frequency $v_{\text {rot }}$ and $r f$ irradiation with an amplitude $v_{1}^{\mathrm{H}}$ in the vicinity of harmonic relationships $v_{1}^{\mathrm{H}}=n v_{\text {rot }}$ where $n=1$ or 2 . Until recently, only the use of very high $r f$ amplitudes (e.g., $v_{1}^{\mathrm{H}} \gg 2 v_{\text {rot }}$ ) made it possible to avoid such interference effects. With the advent of PISSARRO decoupling, the deleterious effects of rotary resonance recoupling can be quenched, notably for $v_{1}^{\mathrm{H}}=2 v_{\text {rot }}$, so that demands on the $r f$ amplitude $v_{1}^{\mathrm{H}}$ are relaxed. Here, we discuss how PISSARRO decoupling benefits from mirror symmetry and phase-shifting. We also show that PISSARRO can cope with the chemical shift anisotropy of protons and with proton-proton dipolar interactions. PISSARRO is most effective for very fast spinning at very high static fields.
\end{abstract}

(c) 2010 Elsevier B.V. All rights reserved.

\section{Introduction}

Heteronuclear dipolar decoupling combined with magic-angle spinning (MAS) allows one to obtain high-resolution solid-state NMR spectra of dilute nuclei such as ${ }^{13} \mathrm{C}$ coupled to abundant spins like protons. Recent progress in the design of MAS probes allows one to achieve routinely spinning frequencies up to $70 \mathrm{kHz}$, which are particularly useful at very high static fields (currently $23.5 \mathrm{~T}$ for proton NMR at $1000 \mathrm{MHz}$.) At very high spinning frequencies and static fields, phase-modulated decoupling methods are to be preferred over continuous wave (CW) decoupling [1]. Yet most methods are prone to interference between spinning at a frequency $v_{\text {rot }}$ and decoupling with an $r f$ nutation frequency $v_{1}^{\mathrm{H}}$, in particular when $v_{1}^{\mathrm{H}}=n v_{\text {rot }}$ with $n=1$ or 2 . These interference effects are due to rotary resonance recoupling $\left(R^{3}\right)$ [2]. Such interference effects can of course be avoided by using very high $r f$ amplitudes $v_{1}^{\mathrm{H}} \gg 2 v_{\text {rot }}$, but this sets high demands on probes and amplifiers.

To overcome this problem, we have developed a heteronuclear decoupling method called 'Phase-Inverted Supercycled Sequence for Attenuation of Rotary Resonance' (PISSARRO) [3]. The PISSARRO pulse sequence comprises phase-alternated pairs of pulses that are repeated $N$ times in two segments featuring mirror symmetry that are phase-shifted through $90^{\circ}$ with respect to each other [3]. This method enables efficient decoupling over a wide range of $r f$ amplitudes, especially at high spinning frequencies where $R^{3}$ interference effects in the vicinity of $n=2$ can be dramatically reduced [4].

\footnotetext{
* Corresponding author at: Ecole Normale Supérieure, Département de Chimie, 24 rue Lhomond, 75005 Paris, France.

E-mail address: Piotr.Tekely@ens.fr (P. Tekely).

1 Current address: Utrecht University, Padualaan 8, 3584 CH Utrecht, The Netherlands.
}

In this study, we shall break down the PISSARRO sequence into different components (see Supplementary Figure S1) and probe their capability to quench $R^{3}$ effects near $n=2$. Eventually, we shall reconstruct the full sequence by combining these elements and show that PISSARRO decoupling near $n=2$ benefits from mirror symmetry and phase-shifting.

\section{Methods and materials}

All spectra of uniformly ${ }^{13} \mathrm{C},{ }^{15} \mathrm{~N}$-labelled L-histidine (L-His) in a partially deprotonated form $[5,6]$ were obtained with a Bruker spectrometer operating at $B_{0}=18.8 \mathrm{~T}\left(800 \mathrm{MHz}\right.$ for $\left.{ }^{1} \mathrm{H}\right)$ and a $1.3 \mathrm{~mm}$ rotor spinning at $v_{\text {rot }}=40$ or $60 \mathrm{kHz}$. The proton carrier frequency was placed to coincide with the isotropic shift of the $\mathrm{H}^{\beta}$ proton. Further experiments with uniformly ${ }^{13} \mathrm{C},{ }^{15} \mathrm{~N}$-labelled L-alanine (L-Ala) were performed at $9.4 \mathrm{~T}\left(400 \mathrm{MHz}\right.$ for $\left.{ }^{1} \mathrm{H}\right)$ and $v_{\text {rot }}=30 \mathrm{kHz}$. The PISSARRO scheme [3] consists of a sequence of $r f$ pulses $\left[\left\{\left(\tau_{x}\right)\left(\tau_{-x}\right)\right\}_{N}\left\{\left(\tau_{-x}\right)\left(\tau_{x}\right)\right\}_{N}\left\{\left(\tau_{y}\right)\left(\tau_{-y}\right)\right\}_{N}\left\{\left(\tau_{-y}\right)\left(\tau_{y}\right)\right\}_{N}\right]$ where the pulse length $\tau$ must be optimized for each spinning frequency $v_{\text {rot }}$ around $\tau=0.3 \tau_{\text {rot }}$ for decoupling near the $n=1$ condition, around $\tau=0.2 \tau_{\text {rot }}$ for decoupling near the $n=2$ condition and around $\tau=0.9 \tau_{\text {rot }}$ or $1.1 \tau_{\text {rot }}$ for very high $r f$ amplitudes (e.g., $v_{1}^{\mathrm{H}} \gg 2 v_{\text {rot }}$ ). We used $N=5$ throughout this work, unless indicated otherwise.

\section{Results and discussion}

\subsection{Experimental results}

The decoupling efficiency of the PISSARRO scheme and its basic module, i.e., a phase-alternated pair of pulses, were probed experimentally at spinning frequencies $v_{\text {rot }}=40$ and $60 \mathrm{kHz}$ over a wide 
range of $r f$ amplitudes $v_{\text {rot }}<v_{1}^{\mathrm{H}}<150 \mathrm{kHz}$. Higher $r f$ amplitudes might damage heat-sensitive samples. The pulse length $\tau$ was optimized to achieve the highest peak height of the $\mathrm{C}^{\beta} \mathrm{H}_{2}$ signal in L-His. Unless otherwise stated, ramped cross-polarization [7] was used to transfer the polarization from protons to carbon-13 nuclei. For each experiment, twelve dummy scans were used to set up a steady state.

PISSARRO's phase-alternated pair of pulses is reminiscent of the XiX method $[8,9]$. As shown in Figure 1, PISSARRO is more efficient than its basic module in the vicinity of $v_{1}^{\mathrm{H}}=80 \mathrm{kHz}$ at $v_{\text {rot }}=40 \mathrm{kHz}$ $(n=2)$ while for $v_{1}^{\mathrm{H}} \geqslant 100 \mathrm{kHz}$ one observes virtually the same performance. For the phase-alternated pair, we found the best decoupling efficiency with $\tau=0.85 \tau_{\text {rot }}$ except for very high $r f$ amplitudes $v_{1}^{\mathrm{H}} \gg 2 v_{\text {rot }}$, where the optimum $\tau$ approaches the recommended pulse length $\tau=2.85 \tau_{\text {rot }}$ of XiX decoupling [9] (see Table 1 in the Supplementary material for details). Note that we focus in this work on the quenching mechanism at the $n=2$ rotary resonance condition and therefore do not refer in general to XiX decoupling for phase-alternated pairs. At $v_{\text {rot }}=60 \mathrm{kHz}$ the performance gap between PISSARRO and phase-alternated pairs widens. PISSARRO shows a good efficiency for $80<v_{1}^{\mathrm{H}}<150 \mathrm{kHz}$. In the range $90<v_{1}^{\mathrm{H}}<130 \mathrm{kHz}$, the $\mathrm{C}^{\beta} \mathrm{H}_{2}$ peak height is about $20-25 \%$ higher with PISSARRO than with phase-alternated pairs and about $10 \%$ higher at $v_{1}^{\mathrm{H}}=140 \mathrm{kHz}$. The $\mathrm{C}^{\alpha} \mathrm{H}$ peak shows a similar pattern, with the same improvement of PISSARRO with respect to phase-alternated pairs. Moreover PISSARRO decoupling gives the same height of the $\mathrm{C}^{\alpha}$ peak at $v_{1}^{\mathrm{H}}=120 \mathrm{kHz}(n=2)$ as at $v_{1}^{\mathrm{H}}=150 \mathrm{kHz}$.

As shown in Figure 1, substantial losses in signal height have to be accepted with low-power PISSARRO or phase-alternated pulse pair decoupling as compared with PISSARRO decoupling close to the $n=2$ condition. For instance at $v_{\text {rot }}=60 \mathrm{kHz}$, decoupling at $v_{1}^{\mathrm{H}}=130 \mathrm{kHz}$ with PISSARRO offers a gain of $30 \%$ in signal height along with a much narrower linewidth (see Figure 1 for signal heights and Figure S6 for linewidths).

The origin of the broadening of the $n=2$ rotary resonance condition observed with the phase-alternated pulse pair irradiation (Figure 1) will be discussed below in a separate section.

\subsection{Numerical simulations}

All simulations were carried out with the SPINEVOLUTION [10] program with and without proton-proton dipolar couplings for the 5-spin fragments $\mathrm{C}^{\alpha} \mathrm{H}^{\alpha} \mathrm{H}^{\beta 1} \mathrm{H}^{\beta 2} \mathrm{H}^{\mathrm{N}}$ and $\mathrm{H}^{\alpha} \mathrm{C}^{\beta} \mathrm{H}^{\beta 1} \mathrm{H}^{\beta 2} \mathrm{H}^{\mathrm{N}}$ of L-serine which have similar $\mathrm{C}^{\alpha} \mathrm{H}^{\alpha} \mathrm{C}^{\beta} \mathrm{H}^{\beta 1} \mathrm{H}^{\beta 2}$ fragments as L-His and other common amino acids. Contributions of the proton chemical shift anisotropy (CSA) were not considered in the simulations discussed in this section. The decoupling efficiency of PISSARRO, the mirror symmetry segment (Figure S1) and the phase-alternated pairs were numerically optimized for best decoupling of $\mathrm{CH}$ and $\mathrm{CH}_{2}$ by independent variation $\left(v_{\text {rot }}-10 \mathrm{kHz}\right)<v_{1}^{\mathrm{H}}<150 \mathrm{kHz}$ and $30<v_{\text {rot }}<70 \mathrm{kHz}$. All simulations used increments of $5 \mathrm{kHz}$ for $v_{1}^{\mathrm{H}}$ and $10 \mathrm{kHz}$ for $v_{\text {rot }}$. For both $\mathrm{CH}$ and $\mathrm{CH}_{2}$ groups, several experimentally observed features could be reproduced in the simulations, in spite of the simplification of the spin systems. Indeed, the simulated data shown in Figure 2a and e confirm that: (i) PISSARRO is more efficient than a simple phase-alternated pair in quenching $R^{3}$ when $v_{1}^{\mathrm{H}} \approx 2 v_{\text {rot }}(n=2)$; (ii) both methods show comparable performance for $v_{1}^{\mathrm{H}} \gg 2 v_{\text {rot }}$; (iii) the 'dips' near recoupling conditions are less pronounced at high spinning frequencies. Moreover, stronger recoupling effects appear for $\mathrm{CH}_{2}$ groups (see Figure S2a and c). In particular, according to experimental observations in the range $60<v_{\text {rot }}<70 \mathrm{kHz}$, the recoupling dips that are seen with the PISSARRO sequence for the $\mathrm{CH}$ group are very flat and narrow (Figure 2e) with only a 5-8\% loss in peak height with

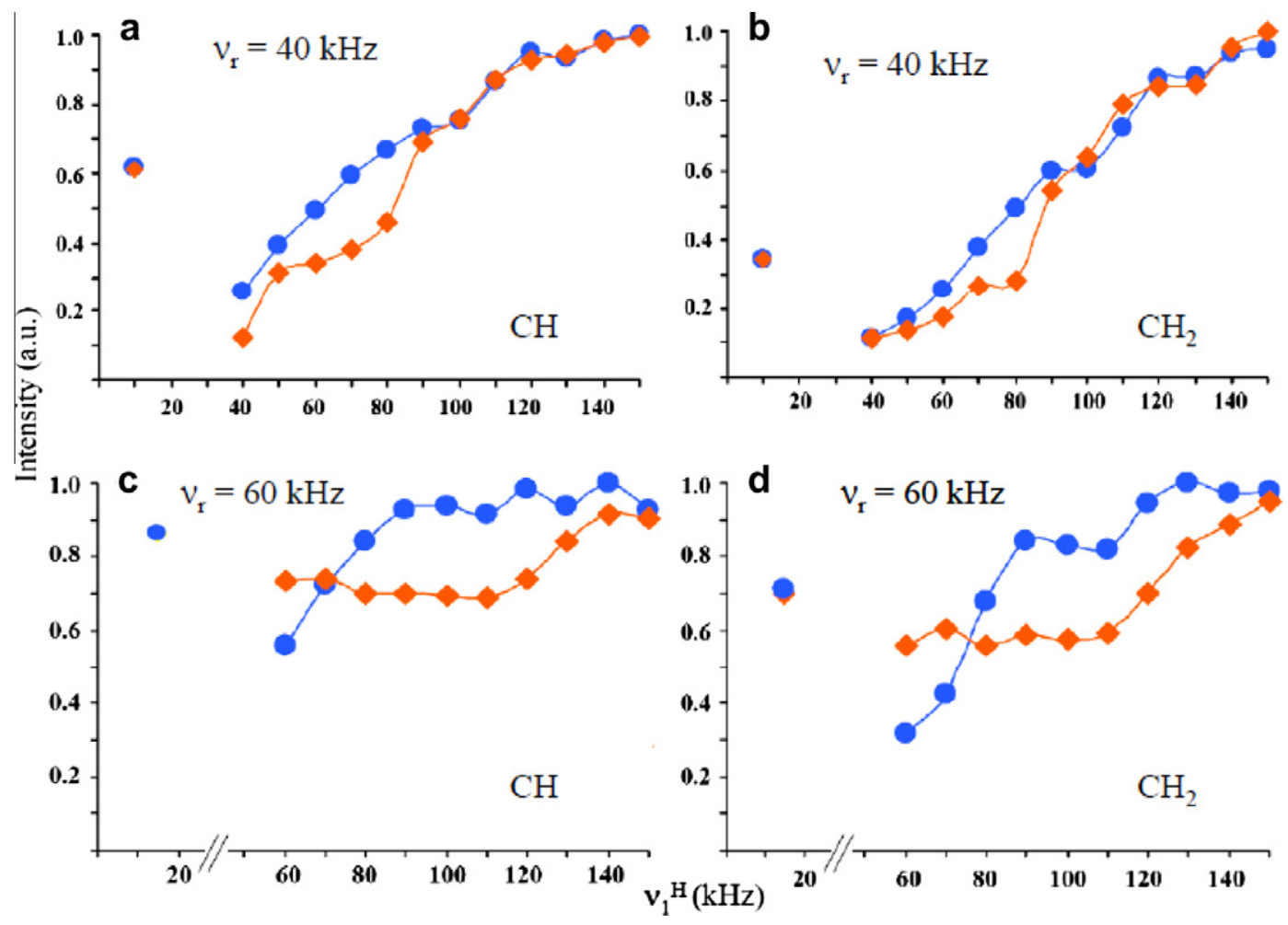

Figure 1. Experimental comparison of PISSARRO (blue circles) and its basic element, i.e., a phase-alternated pulse pair (orange lozenges) at $v_{\text {rot }}=40 \mathrm{kHz}$ (a, b) and $60 \mathrm{kHz}$ (c, d). The peak heights are those of $\mathrm{CH}(\mathrm{a}, \mathrm{c})$ and $\mathrm{CH}_{2}$ (b, d) carbons in L-histidine at $18.8 \mathrm{~T}$ (800 MHz for protons). For each $r f$ amplitude in the range $10<v_{1}^{\mathrm{H}}<150 \mathrm{kHz}<150 \mathrm{kHz}$, the pulse lengths $\tau$ were optimized over the range $0.1 \tau_{\text {rot }}<\tau<4 \tau_{\text {rot }}$ (For interpretation of the references to colour in this figure legend, the reader is referred to the web version of this article.) 

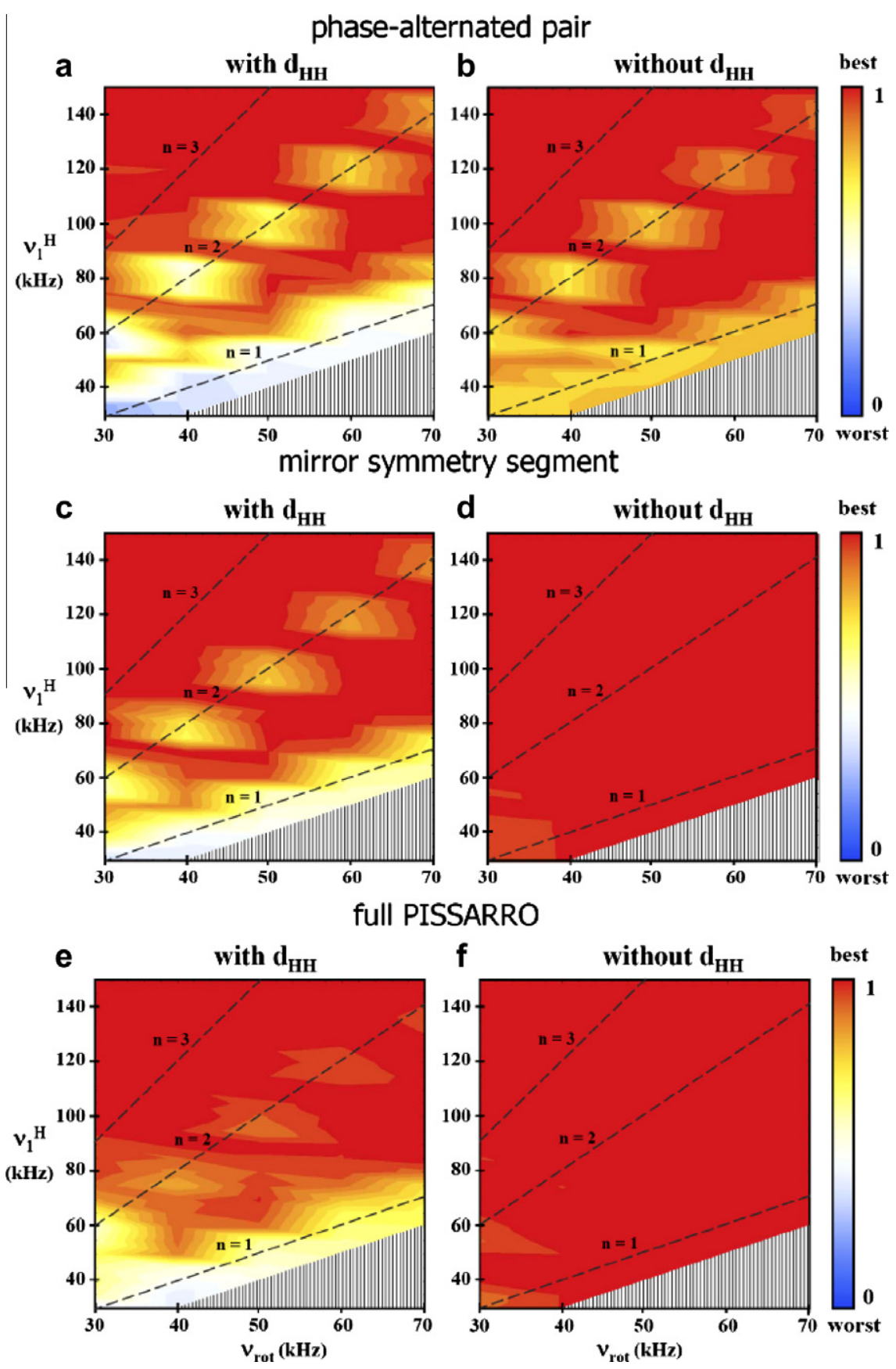

Figure 2. Simulations showing the peak height of the $\mathrm{C}^{\alpha}$ carbon of the $\mathrm{C}^{\alpha} \mathrm{H}^{\alpha} \mathrm{H}^{\beta 1} \mathrm{H}^{\beta 2} \mathrm{H}^{\mathrm{N}}$ fragment of L-serine in a static field of $21.2 \mathrm{~T}$ over a range of $r f$ amplitudes $\left(v_{\text {rot }}-10 \mathrm{kHz}\right)<v_{1}^{\mathrm{H}}<150 \mathrm{kHz}$ and spinning frequencies $30<v_{\text {rot }}<70 \mathrm{kHz}$. with (a, c, e) and without (b, d, f) considering dipolar proton-proton interactions. (a, b) The basic element of PISSARRO, i.e., a phase-alternated pair. (c, d) The first half of PISSARRO, i.e., the mirror symmetry segment. (e, f) Full PISSARRO with $N=5$. The pulse width $\tau$ was optimized in each case. The peak heights were normalised with respected to a simulation using the same $\tau$ while all dipolar interactions were set to zero.

respect to optimal decoupling, while the phase-alternated pair leads to losses of ca. $20-25 \%$ (Figure $2 \mathrm{a}$ ). For $v_{\text {rot }}>50 \mathrm{kHz}$, the phase-alternated pair requires $\mathrm{rf}$ amplitudes that are 10 or $20 \mathrm{kHz}$ higher than for PISSARRO to avoid the recoupling dip, a trend which agrees with the experimental data at $v_{\text {rot }}=60 \mathrm{kHz}$ (Figures 1c and d).

\subsubsection{Role of segments with mirror symmetry}

So far, many experimental observations could be reproduced in simulations, but this does not give much insight into the quenching mechanism of PISSARRO irradiation. Simulations were therefore repeated without proton-proton dipolar couplings $d_{\mathrm{HH}}$ (Figures 2b, f and Figure S1). The removal of $d_{\mathrm{HH}}$ attenuates $R^{3}$ effects for the phase-alternated pair, while for PISSARRO decoupling these effects practically disappear at $v_{\text {rot }}>30 \mathrm{kHz}$.

To identify which features of PISSARRO irradiation contribute to the quenching of $R^{3}$ effects, simulations were run considering solely the first half of the pulse sequence, which we shall refer to as 'mirror symmetry segment' or 'truncated PISSARRO' (Figure S1). As shown in Figure 2d, in the absence of proton-proton dipolar interactions, the mirror symmetry segment turns out to be fully sufficient to yield perfect quenching. This proves the crucial role of mirror symmetry in quenching $R^{3}$ through suppression of heteronuclear dipolar recoupling. Yet, in the presence of proton-proton dipolar interactions (Figure 2c), and in agreement with experimental observations (Figure S3), the truncated version copes less well 
with $R^{3}$ effects than the full PISSARRO sequence. Indeed, as discussed in the next section, the $90^{\circ}$-shifted segments improve the quenching of $R^{3}$ effects by weakening proton-proton dipolar couplings.

\subsubsection{Weakening proton-proton dipolar couplings by $90^{\circ}$ phase- shifted segments}

The comparison between simulations with and without protonproton dipolar couplings $d_{\mathrm{HH}}$ reveals that these couplings hamper the quenching of $R^{3}$ effects in both truncated and full PISSARRO decoupling schemes. Yet, full PISSARRO decoupling clearly performs better, presumably due to its capacity to partially average out $d_{\mathrm{HH}}$. To check this hypothesis, we probed the effect of the $90^{\circ}$ phase-shifted segment on the proton-proton interactions by comparing proton spectra simulated under phase-alternated pairs, truncated PISSARRO and the full PISSARRO scheme. The simulations were done for $\mathrm{C}^{\alpha} \mathrm{H}^{\alpha} \mathrm{H}^{\beta 1} \mathrm{H}^{\beta 2} \mathrm{H}^{\mathrm{N}}$ five spin-system at $v_{\text {rot }}=50 \mathrm{kHz}$ and $v_{1}^{\mathrm{H}}=100 \mathrm{kHz}$ using pulse lengths optimized for heteronuclear decoupling at the $n=2$ condition. All thee irradiation schemes have very different optimal pulse lengths $\tau$ at $v_{1}^{\mathrm{H}} \sim 2 v_{\text {rot }}$ and at high $r f$ amplitudes $v_{1}^{\mathrm{H}} \gg 2 v_{\text {rot }}$. This corresponds to different decoupling mechanisms at and beyond rotary resonance. The optimal pulse length $\tau$ for high $r f$ amplitudes yields minimal non-resonant terms, whereas the optimal quenching pulse length $\tau$ should simultaneously suppress rotary resonance effects and handle non-resonant contributions.

As shown in Figure 3, the peak heights under $r f$ irradiation increase compared with the MAS spectrum simulated in the absence of any $r f$ irradiation. This proves that around $n=2$, all three methods, i.e., full PISSARRO, the mirror symmetry segment and the phase-alternated pair, have some capacity to bring about homonuclear dipolar decoupling. Notably, the full PISSARRO scheme yielded better homonuclear proton-proton decoupling than its truncated version, which reveals the function of the $90^{\circ}$ phase shift of the second half of PISSARRO. Since the quenching of heteronuclear dipolar interactions is similar for PISSARRO and its truncated version in the absence of $d_{\mathrm{HH}}$ terms (Figures $2 \mathrm{~d}$ and $\mathrm{f}$ ), while in

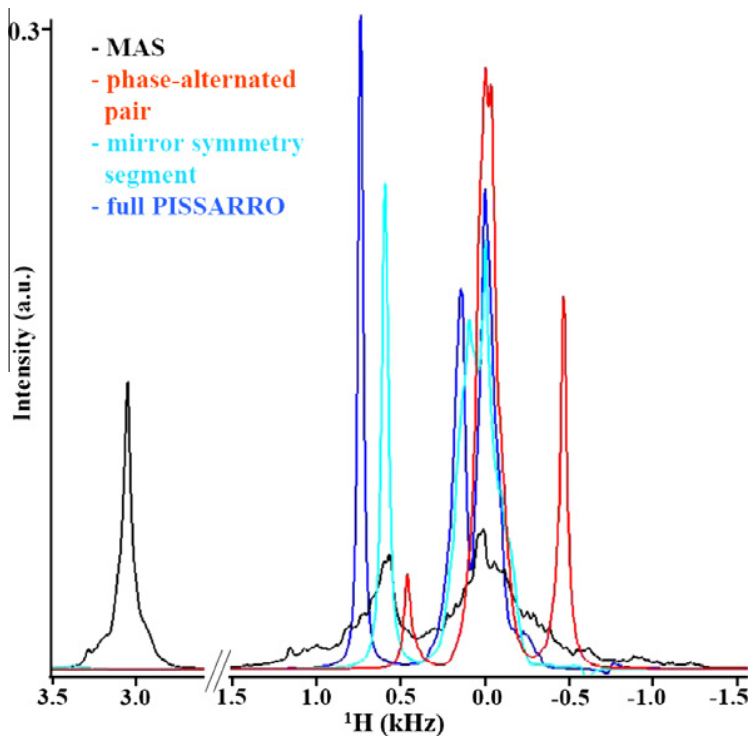

Figure 3. Simulated proton spectra for the $\mathrm{C}^{\alpha} \mathrm{H}^{\alpha} \mathrm{H}^{\beta 1} \mathrm{H}^{\beta 2} \mathrm{H}^{\mathrm{N}}$ fragment of L-serine using basic phase-alternated pairs (red lines), the mirror symmetry segment (light blue lines) and the full PISSARRO sequence (dark blue lines) with $v_{1}^{\mathrm{H}}=100 \mathrm{kHz}$ and $v_{\text {rot }}=50 \mathrm{kHz}$, and without any $r f$ irradiation (black lines). The spectra were normalised to the peak height of a simulation neglecting all dipolar interactions (see Figure S4) (For interpretation of the references to colour in this figure legend, the reader is referred to the web version of this article.). their presence the attenuation of $R^{3}$ effects is superior with the full PISSARRO sequence (Figures $2 \mathrm{c}$ and e), the improved proton-proton decoupling of full PISSARRO must be the reason for its quenching efficiency.

Irradiation by the phase-alternated pair leads to large imageand zero-frequency peaks in proton nutation spectra for all signals that are not exactly on-resonance, whereas spectra simulated under the mirror symmetry segment and full PISSARRO were free of these artefacts (see Figure S4). This is reminiscent of PhaseModulated Lee-Goldburg (PMLG) [11,12] and Decoupling Using Mind Boggling Optimisation (DUMBO) $[13,14]$ methods for homonuclear dipolar proton decoupling. For both methods, the versions employing phase inversions, i.e., two consecutive cycles having a $180^{\circ}$ shift, lead to effective broadband $z$-rotations of the magnetization [15]. In the case of PMLG, the $z$-rotation was shown to be accompanied by a second averaging of homonuclear dipolar terms [16]. For both DUMBO and PMLG $z$-rotation sequences, the spectra were free of image- and zero-frequency peaks. PISSARRO's $180^{\circ}$ shifts have similar consequences.

The efficiency of the phase-alternated pair for homonuclear decoupling is difficult to assess from Figure 3 due to the abovementioned image- and zero-frequency spectral features. Indeed, the high peak intensity of the $\mathrm{H}^{\beta}$ protons at zero-frequency under decoupling with the phase-alternated pair not only results from efficient homonuclear decoupling but also from spin-locked contributions of off-resonance signals. To avoid these complications, the simulations of Figure 3 were repeated by selectively detecting only one of the $\mathrm{H}^{\beta}$ protons which was set on resonance (Figure S5). The simulations show an increased signal intensity of $\mathrm{H}^{\beta}$ under the basic element as compared with the full or truncated PISSARRO sequences. However, this does not translate into good quenching as the phase-alternated pair cannot suppress efficiently the recoupled heteronuclear dipolar interactions around $n=2$ in the absence of homonuclear dipolar proton couplings (Figure $2 \mathrm{~b}$ ).

The simulations also help to understand why the quenching of $R^{3}$ effects near the $n=2$ condition under PISSARRO decoupling improves with increasing MAS frequency. As shown in Figure 2, heteronuclear dipolar contributions at $n=2$ are largely suppressed for $v_{\text {rot }}>30 \mathrm{kHz}$ in the absence of proton-proton couplings. This cannot be the main reason for the gradual improvement with increasing spinning speed. Since proton-proton decoupling by PISSARRO improves with increasing spinning frequencies (Figure 4), this contributes to more efficient quenching of $R^{3}$ effects.

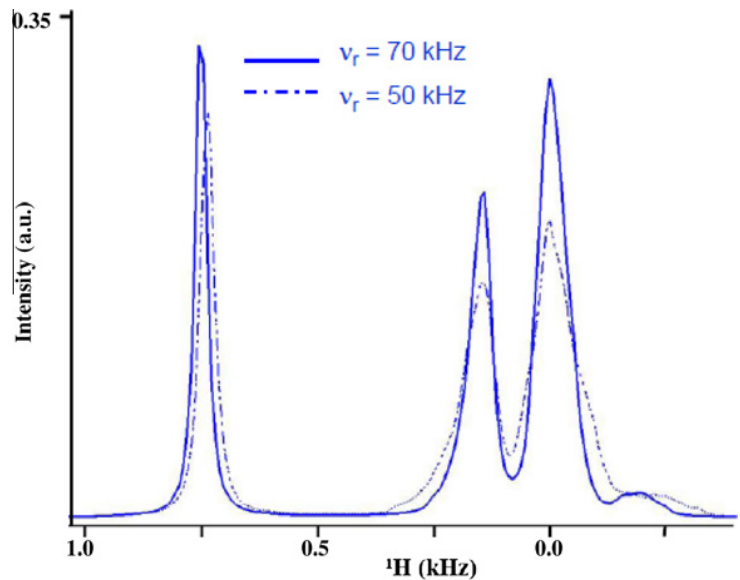

Figure 4. Simulated proton spectra of the $\mathrm{C}^{\alpha} \mathrm{H}^{\alpha} \mathrm{H}^{\beta 1} \mathrm{H}^{\beta 2} \mathrm{H}^{\mathrm{N}}$ fragment of L-serine using full PISSARRO decoupling at $v_{\text {rot }}=50 \mathrm{kHz}$ (dashed-dotted lines) or $70 \mathrm{kHz}$ (continuous lines) and $v_{1}^{\mathrm{H}}=100$ and $140 \mathrm{kHz}$, respectively. All spectra were normalised taking the maximal peak height of Figure $\mathrm{S} 4$ as reference. 
3.2.3. The breadth of the recoupling dips near $n=2$

The breadth of the $R^{3}$ dips near $n=2$ that are observed experimentally for PISSARRO's basic element, i.e., a phase-alternated pair at $v_{\text {rot }}=60 \mathrm{kHz}$ (Figures $1 \mathrm{c}$ and d) could not be reproduced by simulations. Although the addition of further protons in the simulations leads to a slight broadening of the recoupling condition, this turned out to be insufficient to reproduce the experimentally observed breadth. For CW decoupling on the other hand, simulations of an iso-butyl $\left(\mathrm{CH}_{3}\right)_{3} \mathrm{C}$ - system using the SPINEVOLUTION package, showed that the sum of heteronuclear dipolar couplings $d_{\mathrm{CH}}$ is responsible for the broadening of the funnel-shaped dip around $n=2$, while the presence of proton-proton dipolar interactions $d_{\mathrm{HH}}$ leads to a slight narrowing of the recoupling dip. However, for CW decoupling at high spinning speeds, a dramatic decrease of decoupling efficiency due to a residual second order cross-term between ${ }^{1} \mathrm{H}$-CSA and $d_{\mathrm{CH}}$ [17] overshadows this type of broadening.

Indeed, the only interaction that has been disregarded so far in the simulations, apart from scalar contributions, is the proton CSA. Although the decoupling by phase-alternated pulse pair is sufficient to average out a non-resonant cross-term between the proton CSA and the heteronuclear dipolar couplings, other resonant terms involving the proton CSA are reintroduced at the $n=2$ condition. We sought to address the effects of CSA interactions by repeating the simulations for the five spin-system at $v_{\text {rot }}=60 \mathrm{kHz}$ for $\mathrm{rf}$ amplitudes $110<v_{1}^{\mathrm{H}}<150 \mathrm{kHz}$, incorporating CSA tensors for every proton. While the impact on PISSARRO decoupling turned out to be very small, the recoupling dips of the phase-alternated pair featured progressive broadening upon increasing the ${ }^{1} \mathrm{H}$ CSA to $8 \mathrm{ppm}$ (Figure 5a). The curves simulated for a proton CSA of $8 \mathrm{ppm}$ resembled the experimental data remarkably well, showing a wide recoupling dip for the phase-alternated pair (Figure $5 \mathrm{~b}$ ) and the differences between the two methods could be nicely reproduced. For the sake of completeness, we considered a ${ }^{13} \mathrm{C}$ CSA of $25 \mathrm{ppm}$, but this leads to negligible effects for both decoupling methods. Furthermore, the simulations unambiguously demonstrate that proton-proton dipolar interactions $d_{\mathrm{HH}}$ are not only responsible for the depth of the $R^{3}$ dips (Figure 2), but also determine their width (Figure S6). This was confirmed by further expanding the proton network. Indeed, upon addition of a fifth proton, the recoupling dips become more pronounced and broader (Figure 6).

To understand why PISSARRO and its basic phase-alternated pair diverge so strongly at very high spinning frequencies over such a broad range of $r f$ amplitudes, while at slower spinning frequencies significant differences appear only near $n=2$, we need to distinguish the effects of $r f$ amplitudes and pulse lengths $\tau$ on the quenching of proton CSAs. For the phase-alternated pair, if we avoid the $n=2$ condition, we can employ the pulse lengths $\tau=1.9 \tau_{\text {rot }}$ or $2.9 \tau_{\text {rot }}$ or $3.9 \tau_{\text {rot }}$ (or possibly other pulse lengths, if they result in improved performance). For these $\tau$ values, the peak height of the carbon-13 decreases when proton CSAs are included. On the other hand, $\tau=0.85 \tau_{\text {rot }}$ leads to the best decoupling efficiency in the vicinity of $n=2$ and the signal intensity remains remarkably insensitive to proton CSAs. However, the resulting decoupling efficiency cannot compete with what is available with pulse lengths for high $r f$ amplitudes. At a first glance, a similar scenario occurs for PISSARRO decoupling. When using pulse lengths $\tau=0.9 \tau_{\text {rot }}$ or $1.1 \tau_{\text {rot }}$ with high $r f$ amplitudes, the decoupling efficiency is sensitive to proton CSAs, but it remains unaffected for $\tau=0.2 \tau_{\text {rot }}$ used for optimal quenching. However, at very high spinning frequencies, in contrast to the phase-alternated pair, PISSARRO's best quenching pulse length $\tau$ allows better decoupling near the $n=2$ condition than pulse lengths for high $r f$ amplitudes (Figure 5c). This explains why the performances of phase-alternated pairs and PISSARRO decoupling converge for lower spinning
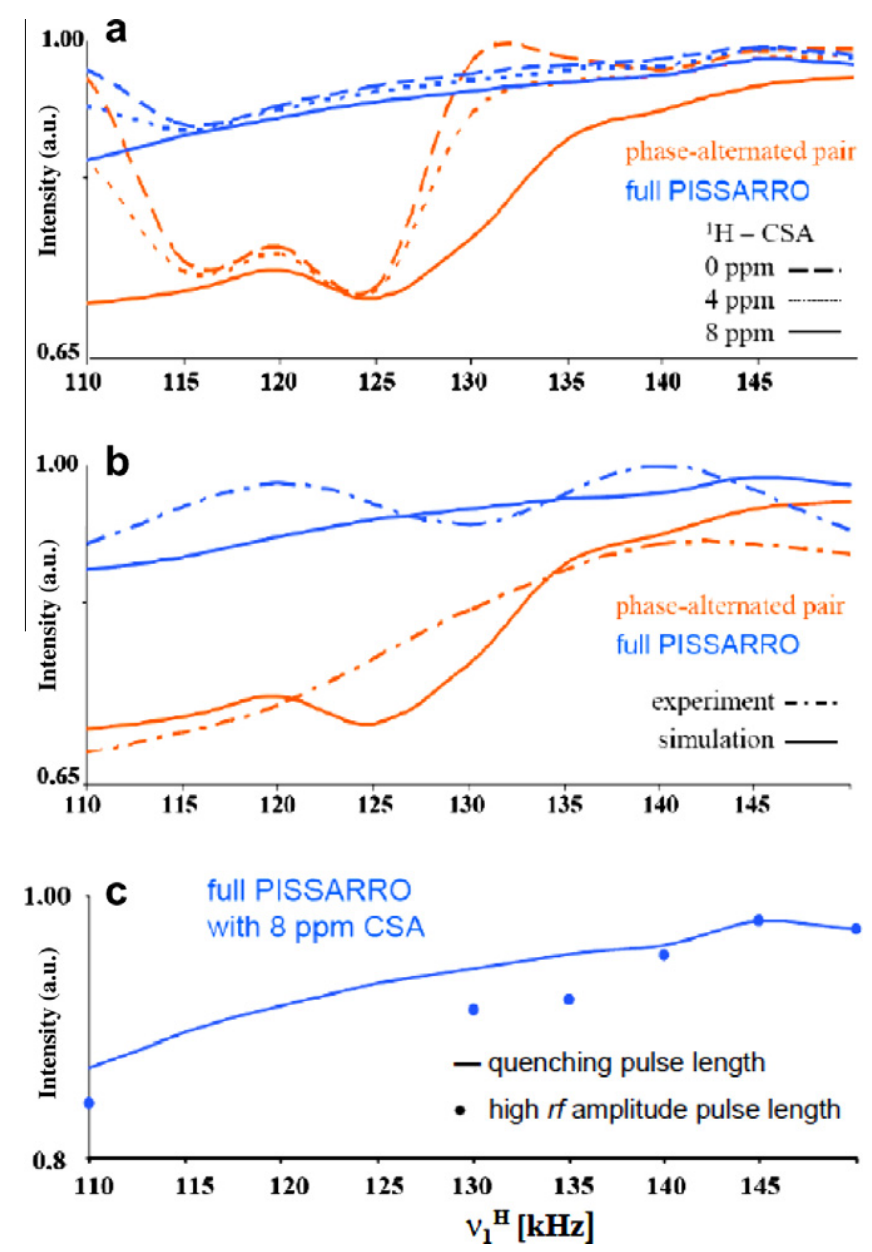

Figure 5. Simulations of the $\mathrm{C}^{\alpha}$ peak height in a $\mathrm{C}^{\alpha} \mathrm{H}^{\alpha} \mathrm{H}^{\beta 1} \mathrm{H}^{\beta 2} \mathrm{H}^{\mathrm{N}}$ fragment of L-serine at $v_{\text {rot }}=60 \mathrm{kHz}$ and $B_{0}=21.2 \mathrm{~T}$ using $110<v_{1}^{\mathrm{H}}<150 \mathrm{kHz}$ in increments of $5 \mathrm{kHz}$. (a) Comparison of basic phase-alternated pairs (orange) and full PISSARRO (blue) without proton CSA (long-dashed lines), or with $\Delta \sigma=4 \mathrm{ppm}$ (short-dashed lines) and $\Delta \sigma=8 \mathrm{ppm}$ (continuous lines) for each proton. The CSA tensors were arbitrarily oriented in the molecular frame, given that their orientation had virtually no influence upon the simulated line-shapes. (b) Comparison of experimental (dasheddotted lines) and simulated data including $\Delta \sigma=8 \mathrm{ppm}$ for each proton (continuous lines) for PISSARRO (blue) and basic phase-alternated pairs (orange). (c) Full PISSARRO decoupling with $\Delta \sigma=8 \mathrm{ppm}$ CSA for each proton using only pulse widths $\tau=0.1967 \tau_{\text {rot }}$ (continuous lines), which is the best pulse length for $R^{3}$ quenching and using the 'high $r f$ amplitude pulse length' $(h \pm 0.1) \tau_{\text {rot }}$ where $h$ is a small integer (blue dots). Since the latter fail near $R^{3}$ conditions, no values are given for $110<v_{1}^{\mathrm{H}}<130 \mathrm{kHz}$ (For interpretation of the references to colour in this figure legend, the reader is referred to the web version of this article.).

frequencies where PISSARRO decoupling using ideal pulse lengths cannot compete with PISSARRO with longer pulse lengths. Consequently, PISSARRO can fully develop its potential in the very fast spinning regime and at very high static fields where other decoupling schemes require much higher $r f$ amplitudes $v_{1}^{\mathrm{H}} \gg 2 v_{\text {rot }}$ to reach the same level of performance [4].

To demonstrate the benefit of very high spinning frequencies for PISSARRO decoupling, we compared peak heights at $v_{\text {rot }}=40$ and $60 \mathrm{kHz}$. PISSARRO was applied with $v_{1}^{\mathrm{H}}=150 \mathrm{kHz}$, using ${ }^{13} \mathrm{C}$ pre-saturation followed by partial recovery and direct carbon excitation, to exclude signal fluctuation due to incomplete longitudinal relaxation. As shown in Figure 7, the peak heights are considerably enhanced at a higher spinning frequency. We found the $C^{\beta}$ peak height to have increased by $\sim 30 \%$, while the $\mathrm{C}^{\alpha}$ signal showed a gain of $60 \%$. It is remarkable that despite the increase of peak height with the spinning frequency, the line-width remains almost unchanged. While peak heights determine the sensitivity, line 

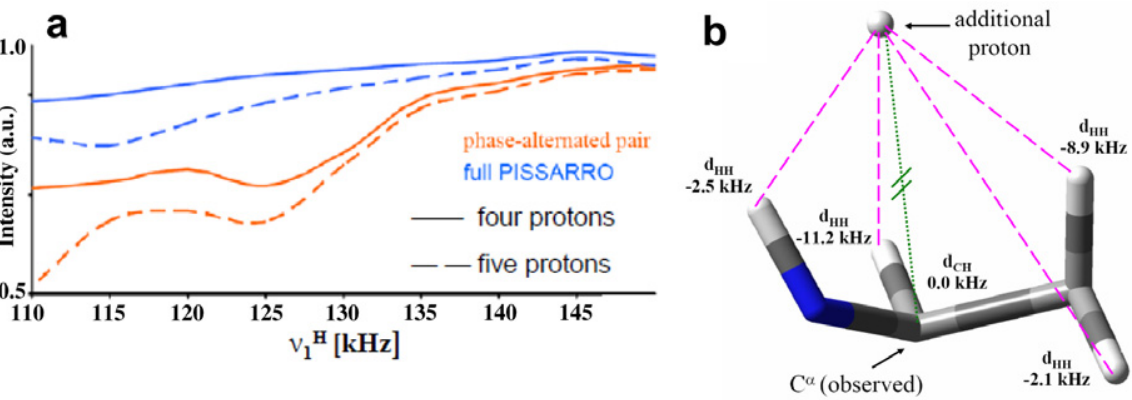

Figure 6. Simulations of the peak height of the $\mathrm{C}^{\alpha}$ signal in a $\mathrm{C}^{\alpha} \mathrm{H}^{\alpha} \mathrm{H}^{\beta 1} \mathrm{H}^{\beta 2} \mathrm{H}^{\mathrm{N}}$ fragment of L-serine with phase-alternated pairs (orange) and full PISSARRO (blue) at $v_{\text {rot }}=60 \mathrm{kHz}$ and $B_{0}=21.2 \mathrm{~T}$ using $110<v_{1}^{\mathrm{H}}<150 \mathrm{kHz}$ in increments of $5 \mathrm{kHz}$ assuming $\Delta \sigma=8 \mathrm{ppm}$ for each proton (continuous lines). In (b), one additional remote proton was included (dashed lines). The heteronuclear coupling $d_{\mathrm{CH}}$ between the observed $\mathrm{C}^{\alpha}$ and the additional proton was neglected (For interpretation of the references to colour in this figure legend, the reader is referred to the web version of this article.).
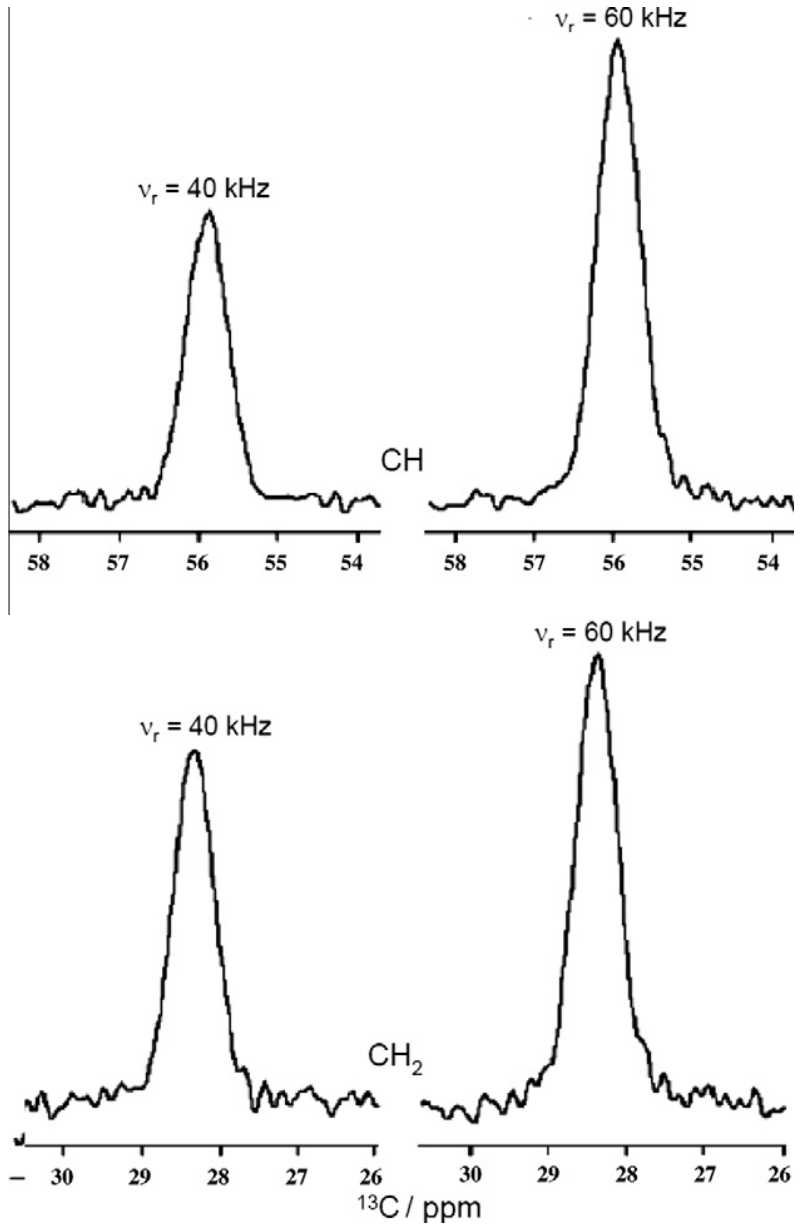

Figure 7. Comparison of the signals of aliphatic $\mathrm{CH}$ and $\mathrm{CH}_{2}$ carbons in L-histidine obtained with full PISSARRO decoupling with $v_{1}^{\mathrm{H}}=150 \mathrm{kHz}$ and $v_{\text {rot }}=40$ or $60 \mathrm{kHz}$ without cross-polarization, i.e., using direct excitation of ${ }^{13} \mathrm{C}$ magnetization.

widths limit the resolution. In the present context, they are not simply inversely proportional to each other. It is therefore important to consider both peak heights and line widths when assessing the performance of various decoupling methods. For on-resonance decoupling, the line-width is independent of the spinning frequency but signal intensity is transferred from sidebands originating from the decoupling scheme [18-20] (see also S7). For PISSARRO, with increasing spinning frequency, signal intensity is transferred from decoupling sidebands to the central line. Note that these sidebands are similar to those we have recently exploited for homonuclear carbon-carbon recoupling [21,22]. Beyond the fact that high spinning frequencies are often necessary at high static fields to prevent residual sidebands and unwanted rotational resonance effects that can lead to line broadening [23] this is a strong argument for the combined use of very high spinning speeds and PISSARRO decoupling. Similar signal enhancements at very high spinning speeds were also reported for homonuclear proton-proton decoupling [24].

\subsubsection{Effect of the block length $N$}

The one remaining feature of the quenching mechanism of PISSARRO decoupling that we wish to comment on is its dependence on the block length $N$. Earlier observations [3] indicated that odd values of $N$ are more efficient than even values in attenuating $R^{3}$ effects near $n=2$. We found that for $\mathrm{C}^{\alpha}$ in L-Ala at $v_{\text {rot }}=30$ and $v_{1}^{\mathrm{H}}=60 \mathrm{kHz}(n=2)$ the signal intensity was $100 \%$ for $N=5,95 \%$ for $N=3,62 \%$ for $N=4$ and $50 \%$ for $N=6$. Simulations for the $C^{\alpha-}$ $\mathrm{H}^{\alpha} \mathrm{H}^{\beta 1} \mathrm{H}^{\beta 2} \mathrm{H}^{\mathrm{N}} 5$-spin cluster under the same conditions but neglecting $d_{\mathrm{HH}}$, also showed a strong attenuation of $R^{3}$ with odd values of $N$. Indeed, we found $100 \%$ relative intensity for $N=5$, followed by $95 \%$ for $N=3,76 \%$ for $N=6$ and $73 \%$ for $N=4$. Re-introducing $d_{\mathrm{HH}}$ reproduces the experimental order with $N=5$ (85\% compared to simulations without $\left.d_{\mathrm{HH}}\right), N=3(82 \%), N=4(69 \%)$ and $N=6$ (68\%). Furthermore, we probed the efficiency of homonuclear proton decoupling for different block lengths $N$. Somewhat surprisingly, the block length $N=5$ turned out to be the least efficient of all four trial values, while $N=3$ yielded the best homonuclear decoupling. This confirms that for the investigated range of spinning frequencies, the efficient suppression of heteronuclear dipolar interactions by mirror symmetry blocks with $N=5$ is a prerequisite for quenching $R^{3}$ effects around $n=2$.

\section{Conclusions}

PISSARRO decoupling has the effect of quenching rotary resonance effects. This has been thoroughly analyzed over a wide range of $r f$ amplitudes. The benefits of very high spinning frequencies and static fields have also been documented. Different constituents of the PISSARRO sequence help to prevent recoupling of heteronuclear dipolar couplings, and to weaken homonuclear proton-proton dipolar couplings, as revealed by numerical simulations. Since the latter interactions are to a large degree averaged out by fast sample spinning, the ability of PISSARRO to quench rotary resonance effects improves with increasing spinning frequency. Since PISSARRO's quenching mechanism is virtually immune against the proton CSA, the method is especially suited for very high magnetic fields. 


\section{Acknowledgments}

Financial support from the Agence Nationale de la Recherche (ANR-09-BLAN-0111-01) and from the Fédération de recherche (FR3050) Très Grands Equipements de Résonance Magnétique Nucléaire à Très Hauts Champs (TGE RMN THC) of the CNRS is gratefully acknowledged.

\section{Appendix A. Supplementary data}

Supplementary data associated with this article can be found, in the online version, at doi:10.1016/j.cplett.2010.12.040.

\section{References}

[1] P. Hodgkinson, Prog. NMR Spectrosc. 46 (2005) 197 (references therein).

[2] T.G. Oas, R.G. Griffin, M.H. Levitt, J. Chem. Phys. 89 (1988) 692.

[3] M. Weingarth, P. Tekely, B. Bodenhausen, Chem. Phys. Lett. 466 (2008) 247.

[4] M. Weingarth, G. Bodenhausen, P. Tekely, J. Magn. Reson. 199 (2009) 238.
[5] B. Henry, P. Tekely, J.J. Delpuech, J. Am. Chem. Soc. 124 (2002) 2025.

[6] C. Gardiennet-Doucet, B. Henry, P. Tekely, Prog. NMR Spectrosc. 49 (2006) 129.

[7] G. Metz, X. Wu, S.O. Smith, J. Magn. Reson. A 110 (1994) 219.

[8] P. Tekely, P. Palmas, D. Canet, J. Magn. Reson. A 107 (1994) 129.

[9] A. Detken, E.H. Hardy, M. Ernst, B.H. Meier, Chem. Phys. Lett. 356 (2002) 298.

[10] M. Veshtort, R.G. Griffin, J. Magn. Reson. 178 (2006) 248.

[11] E. Vinogradov, P.K. Madhu, S. Vega, Chem. Phys. Lett. 314 (1999) 443.

[12] E. Vinogradov, P.K. Madhu, S. Vega, J. Chem. Phys. 115 (2000) 8983.

[13] D. Sakellariou, A. Lesage, P. Hodgkinson, L. Emsley, Chem. Phys. Lett. 319 (2000) 253.

[14] A. Lesage, D. Sakellariou, S. Hediger, B. Elena, P. Charmont, S. Steuernagel, L. Emsley, J. Magn. Reson. 163 (2003) 105.

[15] L. Bosman, P.K. Madhu, S. Vega, E. Vinogradov, J. Magn. Reson. 169 (2004) 39.

[16] M. Leskes, P.K. Madhu, S. Vega, Chem. Phys. Lett. 447 (2007) 370.

[17] M. Ernst, S. Bush, A.C. Kolbert, A. Pines, J. Chem. Phys. 106 (1996) 3387.

[18] G. de Paepe, A. Lesage, L. Emsley, J. Chem. Phys. 119 (2003) 4833.

[19] J. Sachleben, S. Caldarelli, L. Emsley, J. Chem. Phys. 97 (1996) 8947.

[20] J. Sachleben, J. Gaba, L. Emsley, Solid State Nucl. Magn. Reson. 29 (2006) 30.

[21] M. Weingarth, D. Demco, G. Bodenhausen, P. Tekely, Chem. Phys. Lett. 469 2009) 342

[22] M. Weingarth, G. Bodenhausen, P. Tekely, Chem. Phys. Lett. 488 (2010) 10.

[23] D.P. Raleigh, M.H. Levitt, R.G. Griffin, Chem. Phys. Lett. 146 (1988) 71

[24] E. Salager, R.S. Stein, S. Steuernagel, A. Lesage, B. Elena, L. Emsley, Chem. Phys. Lett. 469 (2009) 336. 